\title{
The Microstructure of Ni Layer on Single-Walled Carbon Nanotubes Prepared by an Electroless Coating Process
}

\author{
Weixue Li, ${ }^{1,2}$ Hui Jin, ${ }^{2}$ Yuan Hao, ${ }^{1}$ Tijun Chen, ${ }^{1}$ Jianfeng Dai, ${ }^{1,2}$ and Qing Wang ${ }^{1,2}$ \\ ${ }^{1}$ State Key Laboratory of Gansu Advanced Non-Ferrous Metal Materials, Lanzhou University of Technology, Lanzhou 730050, China \\ ${ }^{2}$ Department of Physics, Lanzhou University of Technology, Lanzhou 730050, China \\ Correspondence should be addressed to Jianfeng Dai, daijf@lut.cn
}

Received 26 April 2010; Accepted 3 September 2010

Academic Editor: Sulin Zhang

Copyright ( 92011 Weixue Li et al. This is an open access article distributed under the Creative Commons Attribution License, which permits unrestricted use, distribution, and reproduction in any medium, provided the original work is properly cited.

\begin{abstract}
The single-walled carbon nanotubes (SWNTs, diameter: $2 \sim 3 \mathrm{~nm}$ ), which were obtained in the suspension of purification solution, with Ni-P coating layers were obtained by an electroless deposition process. The SWNTs before and after coating were characterized by transmission electron microscopy (TEM) and energy dispersive spectrometry (EDS). An Ni-P layer on individual nanotube with thickness of $20 \mathrm{~nm}$ can be obtained after the deposition process. The X-ray diffraction (XRD) and selected area electron diffraction (SAED) analysis of Ni-P SWNTs before and after heat treatment show that the heat treatment caused the transformation of the amorphous Ni-P layer to the nanocrystalline $\mathrm{Ni}-\mathrm{P}$ (crystalline $\mathrm{Ni}$ and $\mathrm{Ni}_{3} \mathrm{P}$ intermetallic compound) layer. The XRD pattern of SWNTs with Ni-P layers after heat treatment revealed that the crystal structures of Ni in plating layer contained: hexagonal closepacked (hcp) structure and face-centered cubic (fcc) structure. The lattice parameters of $\mathrm{Ni}$ (fcc) and $\mathrm{Ni}_{3} \mathrm{P}$ are larger than the bulk's, indicting that the lattice expansion has taken place. However, the lattice parameter of Ni (hcp) has no difference from the bulk's.
\end{abstract}

\section{Introduction}

Since the discovery of carbon nanotubes (CNTs) by Iijima in 1991 [1], much attention has been focused on onedimensional nanomaterials for their outstanding properties, including high length/diameter ratio, low density, and mechanical properties [2, 3]. Significant interest has been recently focused on CNTs-metal composites. However, the bonding of interface between carbon nanotubes and metal is not strong enough. Metal-coated CNTs increase surface active sites to improve interfacial bonding between CNTs and metal matrix. Ni-P layers have been adopted for an increasing number of CNT applications in recent years.

Electroless deposition is an effective approach for the preparation of the CNTs with Ni-P layers. This means that CNTs can be used as reinforcing fibers for metal matrix composites with surface treatment. It will be essential to achieve good interfacial adhesion. The coated layers can serve as medium for adhesion and transferring loads. There have been studies on the morphology of metallic nanoparticles as a function of deposition time in electroless deposition of metal on carbon nanotubes [4].

Multiwall carbon nanotubes (MWNTs) subjected to electroless deposition are produced by CVD which appear to be curved, twisted together, and hard to disperse $[5,6]$. Single-walled carbon nanotubes (SWNTs) adopted in this experiment are straight and easy to disperse. SWNTs with Ni-P coating layers could improve the interfacial bonding between SWNTs and metal matrix and the dispersion of SWNTs in metal matrix.

In order to understand the combining mechanism at the interface, it is necessary to investigate crystal structures under heat treatment. Better thermodynamical stability can be achieved through the nanocrystallization process of the metastable amorphous structure in Ni-P layers, leading to an improvement of the composite properties.

In this study, we prepared SWNTs, which were obtained in the suspension of purification solution, with Ni-P layers by electroless deposition processing. The Ni-P layers are thick and smooth. SWNTs with nanocrystalline Ni-P layers were 
prepared by heat treatment at $400^{\circ} \mathrm{C}$. The samples were characterized with TEM, EDS, SAED, and XRD.

\section{Experimental}

Single-walled carbon nanotubes were prepared by anodic arc discharging plasma. The purification of SWNTs was conducted by using mixed acid $\left(\mathrm{HNO}_{3}: \mathrm{H}_{2} \mathrm{SO}_{4}=1: 3\right)$ to take away amorphous carbon and catalytic nanoparticles. For the purification before further pretreatments for electroless plating, the SWNTs were obtained in the suspension of purification solution, then filtered and washed with deionized water and dried in air at $80^{\circ} \mathrm{C}$. Next, the cleaned SWNTs were sensitized in the aqueous solution containing $\mathrm{SnCl}_{2}+\mathrm{HCl}$ and activated in the aqueous solution containing $\mathrm{PdCl}_{2}+\mathrm{HCl}$ ( the so-called two-step process), followed by washing thoroughly with deionized water. Finally, the pre-treated SWNTs were immersed in the electroless plating bath, which contains $\mathrm{NiSO}_{4}, \mathrm{Na}_{3} \mathrm{C}_{6} \mathrm{H}_{5} \mathrm{O}_{7}$, and $\mathrm{NaH}_{2} \mathrm{PO}_{2}$. Ammonia solution was used as buffer to adjust and maintain $\mathrm{pH}$ value between 8.5 and 8.7. The sample was heat treated under argon atmosphere at $400^{\circ} \mathrm{C}$ for $3 \mathrm{~h}$, where heating rate was $10^{\circ} \mathrm{C} / \mathrm{min}$. The SWNTs before and after coating were characterized with TEM (JEM-2010) and EDS. The Ni-PSWNTs before and after heat treatment were studied using XRD (D8) and SAED.

\section{Results and Discussions}

3.1. Morphological Features of Ni-Coated SWNTs. Figure 1 shows the TEM image of SWNTs after purification, which shows that the CNTs are straight. The diameter of SWNTs is almost $2.6 \mathrm{~nm}$. The purification processing has two effects: taking away the impurities and introducing functional groups on SWNTs surface, such as carboxyl $(-\mathrm{COOH})$ and hydroxyl $(-\mathrm{C}-\mathrm{OH})[7]$. These functional groups change the surface state of SWNTs, which plays an important role in electroless deposition $[8,9]$. The SWNTs have to be oxidized to introduce high density and distributed homogenously functional groups on their surface. This would subsequently lead to a sufficiently high density of adsorbed activator nuclei and a correspondingly distribution of growing $\mathrm{Ni}$ metal nuclei. The growth of Ni would be insufficient to cover the whole tube surface unless the initial density of catalytic sites is very high and homogenous.

Figure 2 shows the representative TEM image of SWNTs with Ni-P coating layers, which clearly indicates that the Ni$\mathrm{P}$ layers coat the tube surface uniformly. The electrolessly deposited Ni-P layers are distributed on the individual tube homogenously. The thickness of the coating layer is more than $20 \mathrm{~nm}$ and as ten times as the semidiameter of the nanotube. The coating thickness can be controlled via reaction time of plating. The layer is symmetric around the SWNTs. Compared to SWNTs produced by CVD, SWNTs adopted in this experiment are straighter, leading to the fewer defects exist on the surface of SWNT. The preparation of tubes with continuous and smooth coating layer has been influenced by two crucial factors: the curvatures of tube $[10,11]$ and the distance between catalytic centers [12].

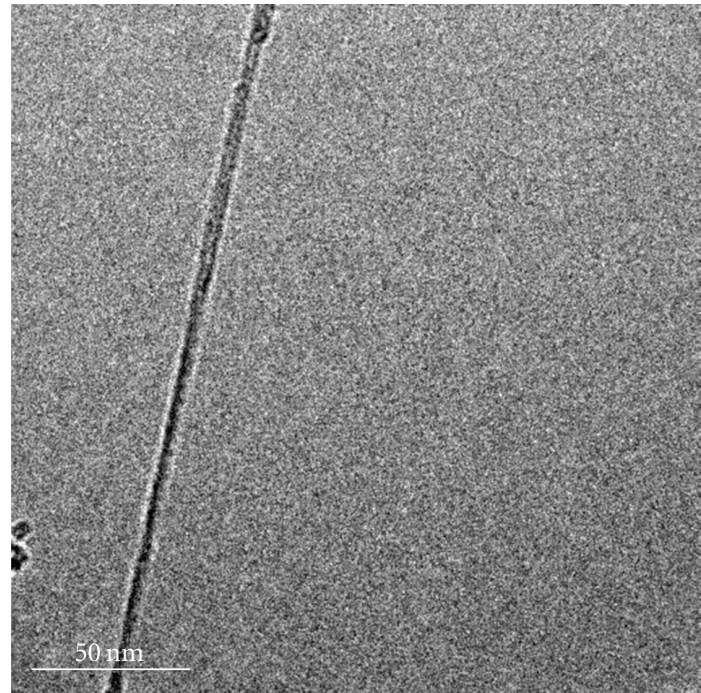

FIGURE 1: TEM image of purified SWNTs.

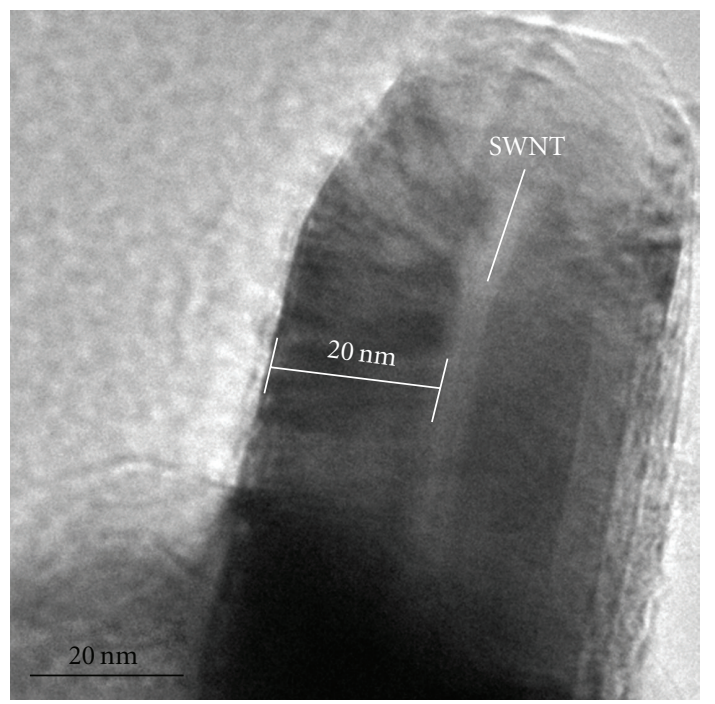

Figure 2: TEM image of the end of as-coated SWNTs.

Figure 3 shows the representative TEM, EDS image of SWNTs with Ni-P layers. The EDS shows the major component in deposited layer is $\mathrm{Ni}$, with a little $\mathrm{P}$ and $\mathrm{Pd}$. The peak of $\mathrm{Cu}$ is due to the grid used in TEM.

\subsection{The Transformation of Crystal Structures of Ni-P Layers.} The electroless deposition $\mathrm{Ni}$ is a metastable and supersaturated alloy consisting of $\mathrm{P}$ atoms between $\mathrm{Ni}$ atoms. The face-centered cubic (fcc) structure of Ni may be maintained within small grains. The structure is essentially amorphous in the regions where the fcc structure cannot be maintained at all [13]. The as-coated SWNTs have been confirmed to be the SWNTs with the amorphous Ni-P layers, and the SWNTs with nanocrystalline Ni-P layers can be prepared by the heat treatment at $400^{\circ} \mathrm{C}$. 


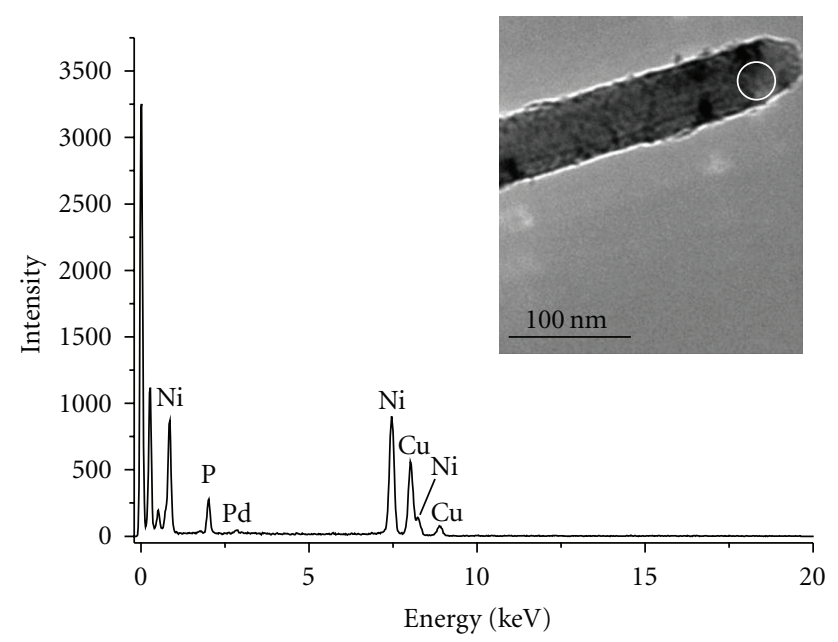

FIgURE 3: TEM image of as-coated SWNTs and EDS, which was selectively taken on the edge of SWNTs with Ni-P layers.

In order to observe the nanocrystallization structures, the SWNTs with Ni-P deposition layers were analyzed by XRD method. In addition to XRD, the SAED analysis was also performed to determine the phase transformation. Figure 4 shows the results.

The XRD pattern of as-coated SWNTs afforded only a broad peak showing the amorphous phase structure $[14,15]$. And the SAED is a broad ring characteristic of an amorphous phase without any trace of crystalline phases. Under heat treatment, the $\mathrm{Ni}$ and $\mathrm{Ni}_{3} \mathrm{P}$ (intermetallic compound) peaks became sharp $[16,17]$. The SAED shows a polycrystalline structural feature, which is in agreement with the results of the XRD analysis. These results have demonstrated that the SWMNs with nanocrystalline Ni-P layer can be prepared by heat treatment at $400^{\circ} \mathrm{C}$ using the SWNTs with amorphous Ni-P layers as the precursors.

\subsection{Microstructure of Ni-P Layers under Heat-Treatment.} Figure 5 shows the part of XRD pattern of SWNTs with Ni-P layers under heat treatment. $2 \theta=\left(40^{\circ}, 56^{\circ}\right)$. There is one sharp and intense peak of $\mathrm{Ni}_{3} \mathrm{P}$ at $2 \theta=41.65^{\circ}$, several weak peaks are observed, and these peaks correspond to the (231), (330), (112), (141), (222) and (132) planes of body-centered tetragonal structure of $\mathrm{Ni}_{3} \mathrm{P}$. The XRD pattern clearly shows that $\mathrm{Ni}$ has two crystal structures in deposition layers after heat treatment: hexagonal close-packed (hcp) structure and face-centered cubic ( $\mathrm{fcc}$ ) structure. The peaks at $2 \theta=45.07^{\circ}$ and at $2 \theta=44.37^{\circ}$ correspond to the (011) plane of $\mathrm{Ni}$ (hcp) and (111) plane of $\mathrm{Ni}$ (bcc), respectively. As we know, there are three crystal structures of Ni: face-centered cubic (fcc), hexagonal close-packed (hcp) and body-centered cubic (bcc). The Ni (hcp) is metastable, but $\mathrm{Ni}$ (hcp) stably exists in the deposition layers under heat treatment.

As shown in Figure 6, there is an obvious phenomenon in XRD pattern. The crystal plane distance calculated from XRD spectrum is different with the bulk's value, which reflects the change of lattice parameter.

For example, $d_{(111)}$ of $\mathrm{Ni}$ (fcc) is $2.04006 \AA$ in Figure 6 and is larger than the bulk's value $d_{(11) 0}=2.01000 \AA$ indicting

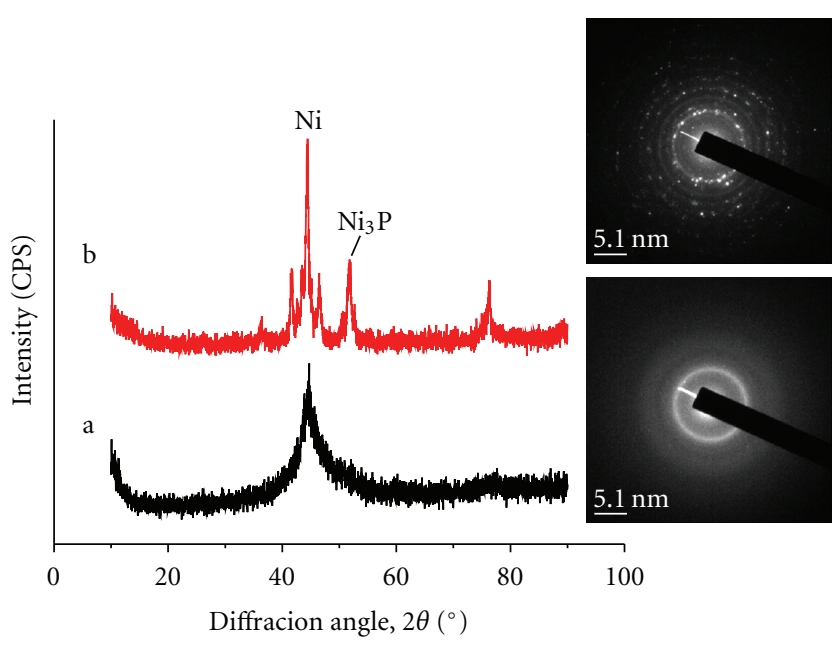

FIGURE 4: XRD and SAED image of SWNTs with Ni-P layers: a ascoated, $\mathrm{b}$ under heat treatment.

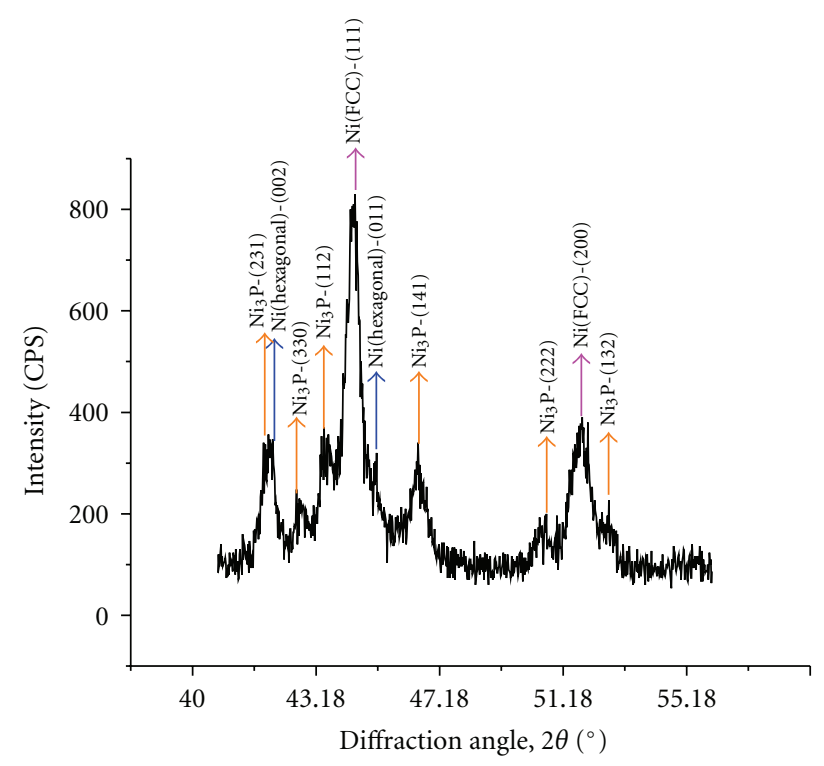

FIGURE 5: Partly of XRD pattern of SWNTs with Ni-P layers under heat treatment.

the emergence of lattice expansion. The rate of the change of crystal plane distance is $\left(d_{(111)}-d_{(111) 0}\right) / d_{(111) 0}=1.5 \%$. And $d_{(231)}$ of $\mathrm{Ni}_{3} \mathrm{P}$ (body-centered tetragonal) is $2.16654 \AA$, but the bulk's value $d_{(231) 0}=2.16100 \AA$. The rate of the change of crystal plane distance is $\left(d_{(231)}-d_{(231) 0}\right) / d_{(231) 0}=0.26 \%$.

While the lattice of $\mathrm{Ni}$ ( $\mathrm{fcc}$ ) and $\mathrm{Ni}_{3} \mathrm{P}$ (bct) has expanded, the lattice of $\mathrm{Ni}$ (hcp) has no change nearly. The change of the lattice parameters of $\mathrm{Ni}$ (hcp) can be ignored.

The change of crystal plane distance shows that the lattice of $\mathrm{Ni}$ ( $\mathrm{fcc}$ ) and $\mathrm{Ni}_{3} \mathrm{P}$ (bct) has expanded. There is a reason as following: the relationship between the lattice parameter and the grain size has been studied by Wei Zhi-Qiang and Lu Ke using the experiment. These studies indicate that the lattice parameter increase significantly with the decrease of the grain size $[18,19]$. So the lattice parameter of nanocrystalline 


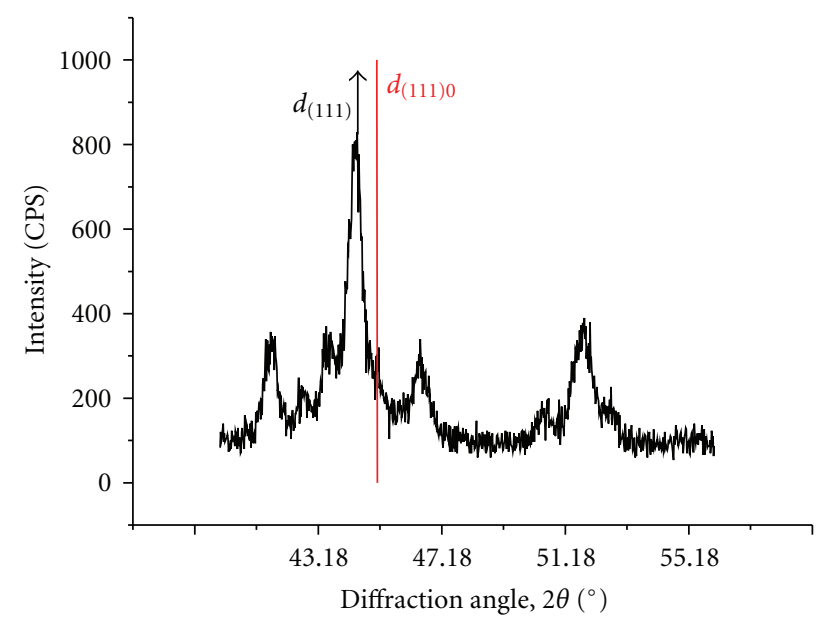

(a)

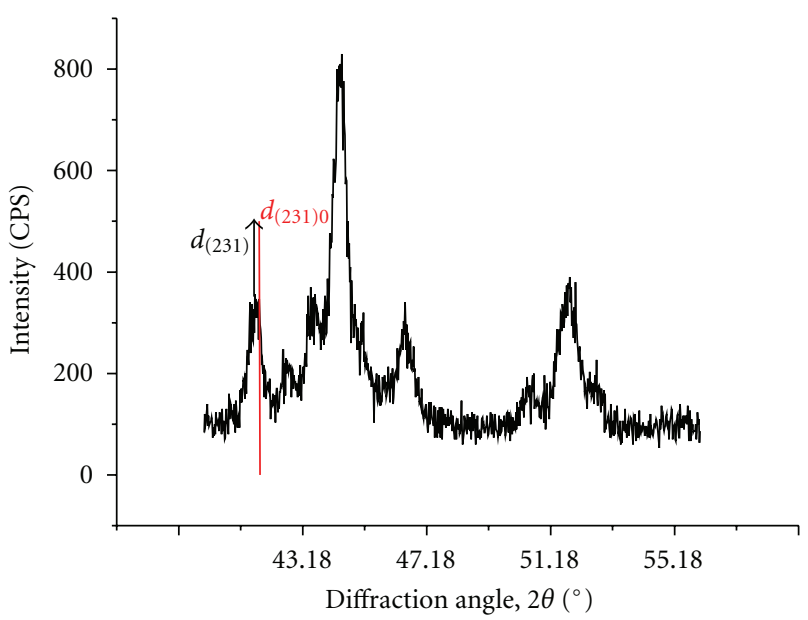

(b)

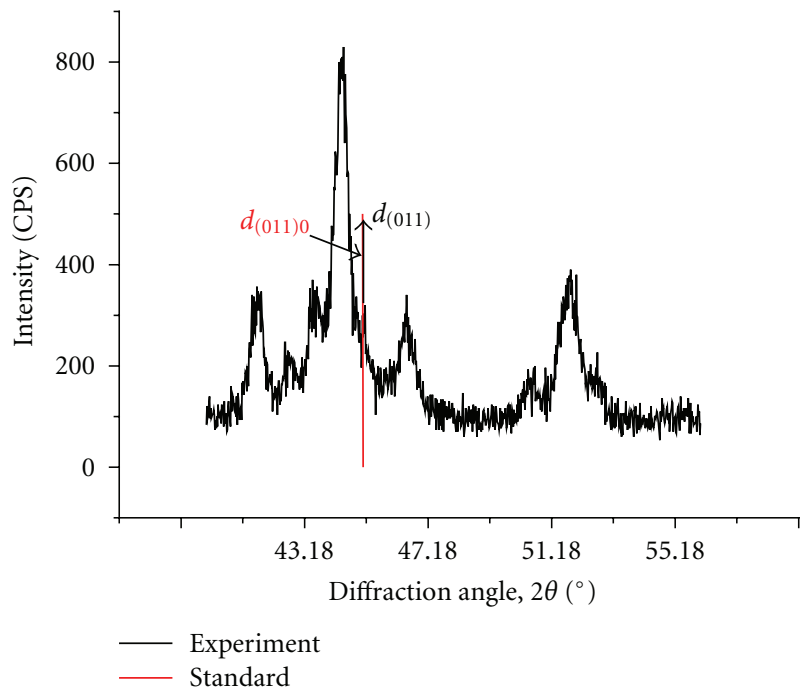

(c)

FIgURE 6: The change of crystal plane distance: a-Ni (fcc); b-Ni ${ }_{3} \mathrm{P} ; \mathrm{c}-\mathrm{Ni}$ (hcp).

in Ni-P layers will increase when they are in nanometer scale due to the grain refinement of nanocrystalline.

\section{Conclusion}

In this paper, the one-dimensional SWNTs with continuous and dense coating layer have been successfully synthesized by an electroless deposition process. The thickness of the coated layer is more than $20 \mathrm{~nm}$. It has been found that the heat treatment can convert the amorphous Ni-P coating layers into the nanocrystalline $\mathrm{Ni}-\mathrm{P}\left(\mathrm{Ni}\right.$ and $\mathrm{Ni}_{3} \mathrm{P}$ ) layers. Our results show that the lattice of $\mathrm{Ni}$ (fcc) and $\mathrm{Ni}_{3} \mathrm{P}$ (bct) has expanded but the rates of expansionary not equal. At the same time, the lattice of $\mathrm{Ni}$ (hcp) has contracted and the the change could be ignored.

\section{Acknowledgments}

This work is financially supported by the Natural Science Foundation of China (no. 50873047) and the Natural Science Foundation of Gansu Province (no. 3ZS062-B25020), China.

\section{References}

[1] S. Iijima, "Helical microtubules of graphitic carbon," Nature, vol. 354, no. 6348, pp. 56-58, 1991.

[2] Z. W. Pan, S. S. Xie, L. Lu et al., "Tensile tests of ropes of very long aligned multiwall carbon nanotubes," Applied Physics Letters, vol. 74, no. 21, pp. 3152-3154, 1999.

[3] M. S. Dresselhaus, G. Dresselhaus, and P. C. Eklund, Science of Fullerenes \& Carbon Nanotubes, Academic Press, San Diego, Calif, USA, 1996. 
[4] J. H. Byeon and J. Hwang, "Morphology of metallic nanoparticles as a function of deposition time in electroless deposition of metal on multi-walled carbon nanotubes," Surface and Coatings Technology, vol. 203, no. 1-2, pp. 357-363, 2008.

[5] Z. P. Huang, J. W. Xu, Z. F. Ren, J. H. Wang, M. P. Siegal, and P. N. Provencio, "Growth of highly oriented carbon nanotubes by plasma-enhanced hot filament chemical vapor deposition," Applied Physics Letters, vol. 73, no. 26, pp. 3845-3847, 1998.

[6] P. M. Ajayan and O. Z. Zhou, "Applications of carbon nanotubes," Carbon Nanotubes, vol. 80, pp. 391-425, 2001.

[7] C.-S. Chen, X.-H. Chen, Z. , W.-H. Li, L.-S. Xu, and B. $\mathrm{Yi}$, "Effect of multi-walled carbon nanotubes as reinforced fibres on tribological behaviour of Ni-P electroless coatings," Diamond and Related Materials, vol. 15, no. 1, pp. 151-156, 2006.

[8] P. Serp, M. Corrias, and P. Kalck, "Carbon nanotubes and nanofibers in catalysis," Applied Catalysis A, vol. 253, no. 2, pp. 337-358, 2003.

[9] M. A. Fraga, E. Jordão, M. J. Mendes, M. M. A. Freitas, J. L. Faria, and J. L. Figueiredo, "Properties of carbon-supported platinum catalysts: role of carbon surface sites," Journal of Catalysis, vol. 209, no. 2, pp. 355-364, 2002.

[10] Q. Li, S. Fan, W. Han, C. Sun, and W. Liang, "Coating of carbon nanotube with nickel by electroless plating method," Japanese Journal of Applied Physics, Part 2, vol. 36, no. 4, pp. L501-L503, 1997.

[11] X. Chen, J. Xia, J. Peng, W. Li, and S. Xie, "Carbon-nanotube metal-matrix composites prepared by electroless plating," Composites Science and Technology, vol. 60, no. 2, pp. 301-306, 2000.

[12] F. Z. Kong, X. B. Zhang, W. Q. Xiong et al., "Continuous Nilayer on multiwall carbon nanotubes by an electroless plating method," Surface and Coatings Technology, vol. 155, no. 1, pp. 33-36, 2002.

[13] L. M. Ang, T. S. A. Hor, G. Q. Xu, C. H. Tung, S. P. Zhao, and J. L. S. Wang, "Decoration of activated carbon nanotubes with copper and nickel," Carbon, vol. 38, no. 3, pp. 363-372, 2000.

[14] F. Wang, S. Arai, and M. Endo, "The preparation of multiwalled carbon nanotubes with a Ni-P coating by an electroless deposition process," Carbon, vol. 43, no. 8, pp. 1716-1721, 2005.

[15] Á. Révész, J. Lendvai, J. Lóránth, J. Pádár, and I. Bakonyi, "Nanocrystallization Studies of an Electroless Plated Ni-P Amorphous Alloy," Journal of the Electrochemical Society, vol. 148, no. 11, pp. C715-C720, 2001.

[16] K. G. Keong, W. Sha, and S. Malinov, "Crystallization and phase transformation behaviour of electroless nickelphosphorus deposits with low and medium phosphorus contents under continuous heating," Journal of Materials Science, vol. 37, no. 20, pp. 4445-4450, 2002.

[17] Á. Révész, J. Lendvai, Á. Cziráki, H. H. Liebermann, and I. Bakonyi, "Formation of nanocrystalline phases during decomposition of amorphous Ni-P alloys by continuous linear heating," Zeitschrift für Metallkunde, vol. 92, no. 5, pp. 483$488,2001$.

[18] Z.-Q. Wei, T.-D. Xia, J. Wang, Z.-G. Wu, and P.-X. Yan, "Lattice expansion of Ni nanopowders," Acta Physica Sinica, vol. 56, no. 2, pp. 1004-1008, 2007.

[19] L. Ke and X.-Z. Haoyue, "Lattice expansion in nanocrystalline pure Ni," Acta Metallurgica Sinica, vol. 431, no. 2, pp. A74A78, 1995. 

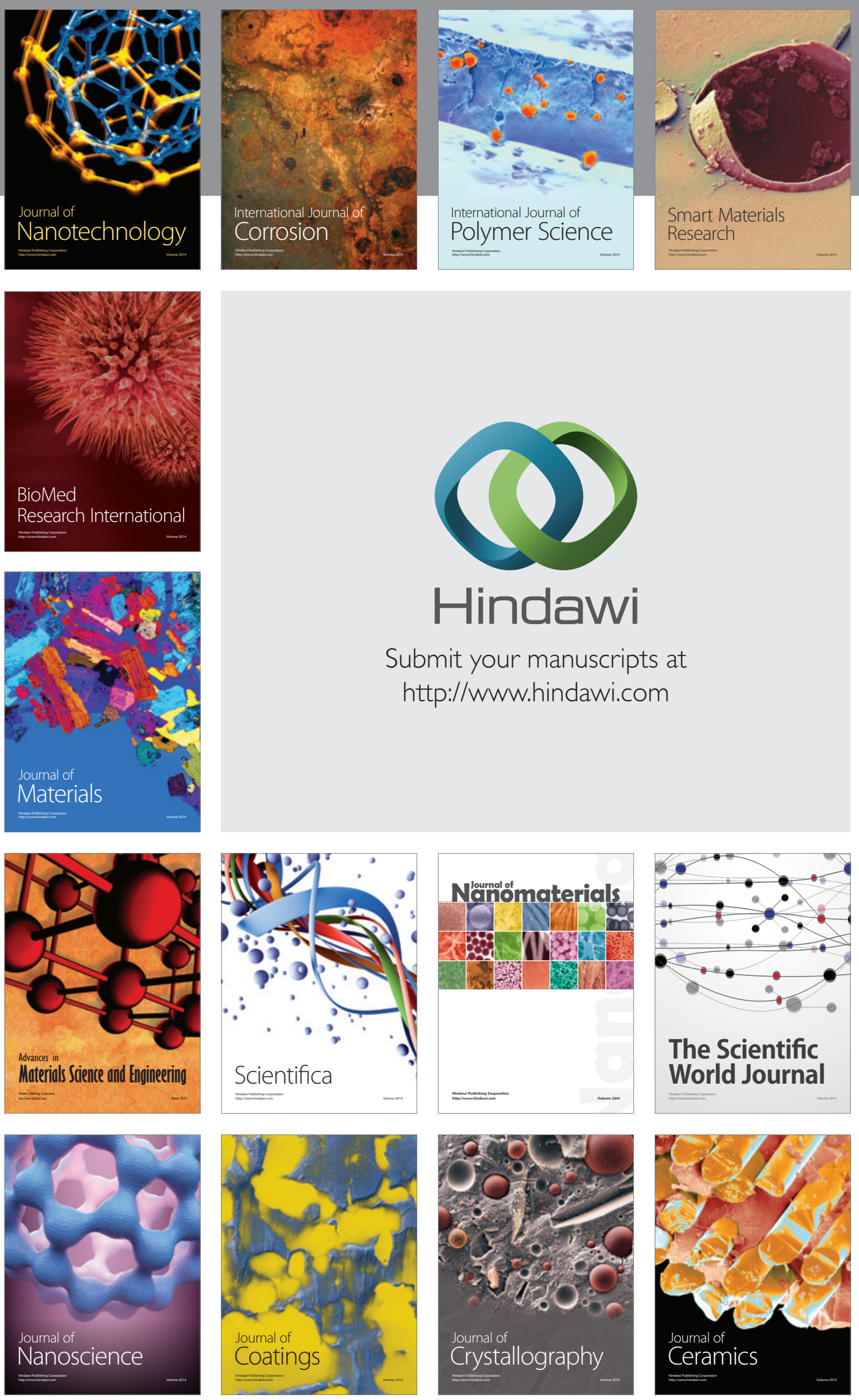

The Scientific World Journal

Submit your manuscripts at

http://www.hindawi.com

\section{World Journal}

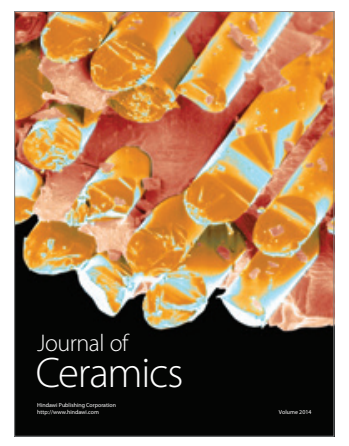

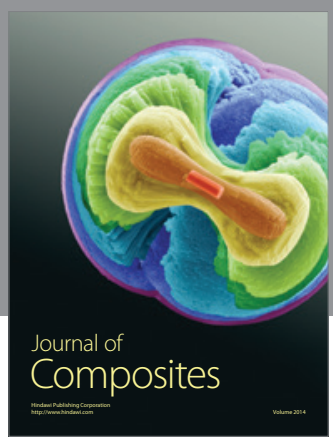
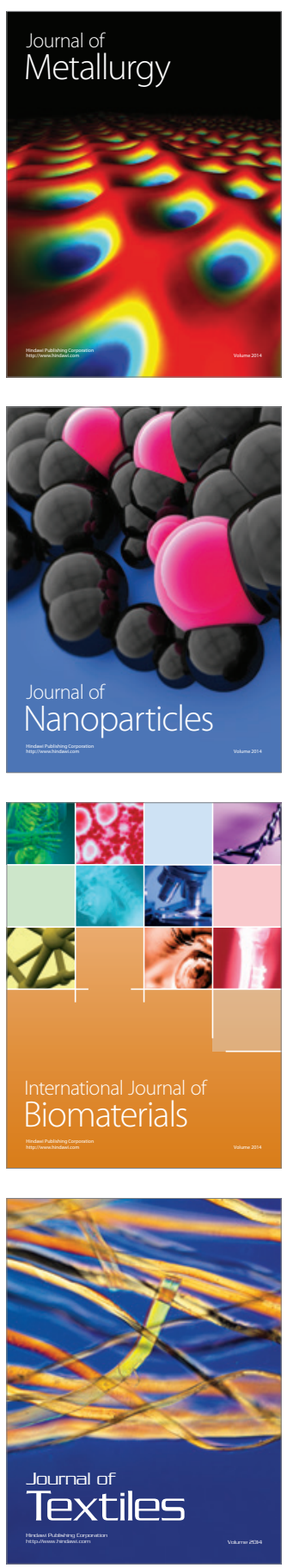